\title{
A study of clinical and laboratory profiles of dengue fever in children
}

\author{
Anand R. ${ }^{1}$, Selvakumar L. ${ }^{2}$, Sagayaraj B. ${ }^{3}$, Sujatha B. ${ }^{4}$, Porchelvan ${ }^{5}$ \\ ${ }^{1}$ Dr. Anand R, ${ }^{2}$ Dr. Selva Kumar L, ${ }^{3}$ Dr. Benjamin Sagayaraj, ${ }^{4}$ Dr. Balamma Sujatha, all above authors are affiliated with \\ Department of Paediatrics, Saveetha Institute of Medical and Technical Sciences. Chennai, Tamil Nadu, ${ }^{5}$ Dr. Porchelvan, \\ Department of Community Medicine, Saveetha Institute of Medical and Technical Sciences. Chennai, Tamil Nadu, India.
}

Corresponding Author: Dr. Anand R, Department of Paediatrics, Saveetha Institute of Medical and Technical Sciences. Chennai, Tamil Nadu. E-mail: anand160988@gmail.com

\begin{abstract}
Background: Early recognition of characteristic clinical and laboratory parameters of is importantto facilitate prompt diagnosis andtreatmentfor a better outcome, particularly in dengue burden countries. Methods: Children aged less than 15 years who sought medical care in the pediatric department of Saveetha medical college, diagnosed as Probable Dengue/Dengue fever/ dengue with warning sign/severe dengue according to standard guidelines during the study period (July 2017 - December 2017) comprised the study sample. Results: A total of 61 cases were enrolled, of these 36 were boys. The mean age was 6.49 years. Only five patients had severe dengue. Fever (96.7\%), loss of appetite (59\%), cough and running nose (33\%) were common symptoms noted and itching (37.7\%) was common during recovery. Only $11.4 \%$ of children had bleeding manifestation. Hepatomegaly was noticed in 35 children. Five children went into shock, 2 of whom had hypotension. NS1 was positive in $49 \%$, IgM in $36 \%$, and $\operatorname{IgG}$ in $13 \%$. NS1 positivity rate on day 4 , day 5 and day 6 of illness were $62.5 \%, 38.4 \%$ and $18.1 \%$ respectively. Conclusion: Dengue fever is common during monsoon season, and the course and severity are highly variable. Though the manifestations of dengue are similar to other viral infections, morbidity and mortality are more, thus requiring early diagnosis. As observed in our study the presence of prodromal respiratory symptoms does not preclude the diagnosis of dengue. There should be a high index of suspicion of co-infection, notablymalaria and scrub typhus, if there is an unusual persistence of fever.
\end{abstract}

Keywords: Dengue fever, Severe dengue, Co-infection

\section{Introduction}

Day-biting Aedesaegypti and Aedesalbopictus transmitsone of four serotypes of dengue viruses (DEN1-4) of the Flaviviridae family to cause dengue fever. While the primary infection in human has often been in apparent, secondary infection by heterologous types frequently causes severe dengue [1]. Dengue is now endemic in over 100 countries, resulting in $40 \%$ of the world's population living mostly in urban and semiurban settings being at risk for dengue.

Although there is no specific treatment for dengue, careful hemodynamic and hematologic parameters monitoring, volume repletion and close observation for the signs of severe disease can reduce dengue' smorbidity and mortality [2]. Dengue virus transmission is ubiquitous with the highest risk in Asia (70\%), Africa (16\%) and South American countries (14\%) of which

Manuscript received: $10^{\text {th }}$ April 2018

Reviewed: $20^{\text {th }}$ April 2018

Author Corrected: $25^{\text {th }}$ April 2018

Accepted for Publication: $30^{\text {th }}$ April 2018 over half occurred in Brazil and Mexico [3]. India alone contributes $14 \%$ of the global burden which is mainly due to densely populated areas that serve as ideal circumstances for the dengue borne mosquitoes to breed and spread disease [4-6].

Dengue is the leading cause of childhood morbidity and mortality globally than any other vector-borne viral disease [7]. In the last few decades, the number of dengue cases has gradually increased in India. Malaria and dengue were the most common causes of fever among hospitalised patients with acute undifferentiated fever [8].

Various factors like change in temperature and precipitation [4], nutritional status of overweight and undernutrition $[9,10]$, inadequate knowledge and preventive measures among the less privileged population about dengue fever attributes to this scenario [11]. 
The lack of effective and safe dengue vaccine is also a major factor for increased morbidity and mortality among high-risk population given the lack of denguespecific antiviral agents. The recent studies [12,13] show that the overall efficacy of CYD-TDV vaccine is $54 \%$ (40-64), while serotype-specific efficacy is $77 \%$ (66-85) for DENV4, 75\% (65-82) for DENV3, 50\% (36-61) for DENV1, and 34\% (14-49) for DENV2.15\% (174-74) vaccine efficacy for unknown serotype.

Although CYD-TDV vaccine is effective and immunogenic in children, its reduced efficacy against DENV2, which is known for causing severe dengue infection and dengue outbreaks, cause for serious concern. This review aims to address common clinical and laboratory parameters to facilitate early diagnosis of dengue fever, to determine predictors of severe dengue and to initiate appropriate treatment for a better outcome

\section{Material and Methods}

Place of study- Department of Pediatric, Saveetha medical college, Chennai

Type of study- Retrospective observational study

Sampling method- Consecutive sampling

Inclusion criteria- All patients aged less than 15 years who sought medical care in the pediatric department of
Saveetha medical college, diagnosed as Probable Dengue/Dengue fever/ dengue with warning sign/severe dengue according to standard guidelines [14]

Exclusion criteria- Children with preexisting haematological illnesses

Sample collection- For each patient, basic demographics (name, age, sex, address) was collected. Presenting complaints (duration of fever, myalgia, headache, abdominal pain, vomiting, bleeding manifestations, rash and petechiae) and examination findings (vitals, Hess test, pallor, organomegaly, urine output, signs of fluid retention, circulatory failure)were documented.

Serial laboratory investigations (haemoglobin, total leukocyte count, haematocrit and platelet count) and investigations like Serum glutamic pyruvic transaminase (SGPT), serum glutamic oxalic acid (SGOT) and serum albumin were recorded.

Radiological investigations like ultrasound abdomen and chest X-ray was taken into consideration if available. Details of co-infections/ alternate diagnosis considered at admission/discharge were also recorded.

Statistical method- Statistical analysis was done using SPSS (statistical package for social sciences) software.

\section{Results}

A total of 61 cases were enrolled in the months September, October and November, of these 36 were boys and 25 girls. Most of the admissions were in October (61\%). There were 14, 37 and 10 admissions in September, October and November respectively. The mean age was 6.49 years; the youngest child isten months old. Of the total cases, the maximum number of admissions was in October (38) which constituted to about $\mathbf{6 1} \%$ of the total patients. Only five patients were diagnosed to have severe dengue.

Table-1: Demographic distribution

\begin{tabular}{|c|c|c|c|}
\hline Age & Boys & Girls & Total \\
\hline 7-12 months & 0 & 1 & 6 \\
\hline $1-2$ years & 1 & 5 & 17 \\
\hline 2-5 years & 8 & 9 & 34 \\
\hline $6-12$ years & 24 & 10 & 3 \\
\hline $13-15$ years & 3 & 0 & 6 \\
\hline
\end{tabular}

Fever was the most common symptom seen in $96.7 \%$ of children among which $40(65.6 \%)$ had an only mild fever. Only $\mathbf{1 3} \%$ had temperature $>104^{\circ} \mathrm{F}$. The next most common symptom was a loss of appetite present in $\mathbf{3 6}$ (59\%). Majority of patients did not have any prodromal symptoms; However, 20 (33\%) children had cough and runny nose.

A headache was present in $26(\mathbf{4 3 \%})$ patients, and $33(54 \%)$ had vomiting. During the recovery phase, $23(37.7 \%)$ children had itching. Loose stools were infrequently present in 7 children (11.5\%). 
Table-2: Clinical features

\begin{tabular}{|c|c|}
\hline Clinical Parameters & $\mathbf{n}(\mathbf{\% )}$ \\
\hline Fever & $59(96.7)$ \\
\hline Chills and Rigor & $13(21.3)$ \\
\hline Headache & $26(42.6)$ \\
\hline Rash & $13(21.3)$ \\
\hline Flushed Look & $25(41.0)$ \\
\hline Hess Test Positivity & $18(29.5)$ \\
\hline Vomiting & $33(54.1)$ \\
\hline Loose Stools & $7(11.5)$ \\
\hline Arthralgia & $26(42.6)$ \\
\hline Myalgia & $29(47.5)$ \\
\hline Anorexia & $36(59.0)$ \\
\hline Abdominal Pain & $28(45.9)$ \\
\hline Cough or Runny nose & $20(32.7)$ \\
\hline Itching & $23(37.7)$ \\
\hline
\end{tabular}

On clinical assessment, $40 \%$ of children had rash and flushed skin. Hess test was positive in $\mathbf{2 9 . 5 \%}$ of the children. Only $\mathbf{1 1 . 4 \%}$ of children had bleeding manifestation. Hepatomegaly noticed in 35 children and hepato splenomegaly in $\mathbf{2}$ children. Most children had heart rate within normal limits, and14.7\% had brady cardia. Five children went into shock, 2 of whom had hypotension.

NS 1 was positive in 30 children (49\%), IgM in 36\% and IgG in $\mathbf{1 3} \%$. NS1 positivity rate on day 4, day 5 and day 6 of illness was $\mathbf{6 2 . 5 \%}, \mathbf{3 8 . 4 \%}$ and $\mathbf{1 8 . 1} \%$ respectively. SGOT elevation was noticed in $\mathbf{2 3}$ children. Most children had mildmoderate thrombocytopenia (59\%). Only $\mathbf{2 6 . 2} \%$ had severe thrombocytopenia. The mean day of platelet recovery was between day 7 and 9 of illness. PCV change of $>20 \%$ was found in $13(\mathbf{2 1 . 3 \%})$ patients and $35(\mathbf{5 7 . 4 \% )}$ children had $<$ $20 \%$ rise in PCV. 24 (39.3\%) of the children had some degree of anaemiaand more than 2/3rd of the patients had leucopenia (44).

Table-3: Clinical Signs

\begin{tabular}{|c|c|}
\hline Clinical Signs & N (\%) \\
\hline Bleeding Manifestation & $7(11.5)$ \\
\hline Oedema & $9(14.8)$ \\
\hline Tachycardia & $4(6.6)$ \\
\hline Bradycardia & $9(14.8)$ \\
\hline Tachypnea & $4(6.6)$ \\
\hline Oliguria & $4(6.6)$ \\
\hline Compensated Shock & $3(4.9)$ \\
\hline Hypotensive Shock & $2(3.3)$ \\
\hline Hepatomegaly & $35(57.4)$ \\
\hline Hepatosplenomegaly & $2(3.3)$ \\
\hline
\end{tabular}

Table-4: Laboratory values

\begin{tabular}{|c|c|}
\hline Laboratory Values & $\mathrm{n}(\%)$ \\
\hline Anaemia & $24(39.3)$ \\
\hline Thrombocytopenia & $57(93.4)$ \\
\hline Leucopenia & $44(72.1)$ \\
\hline Hyponatremia & $5(8.2)$ \\
\hline Hypoalbuminemia & $5^{*}(\mathrm{~N}=49)$ \\
\hline Ns1 Antigen Positivity & $30(49.2)$ \\
\hline Serology (I) IgM Positive & $14(23)$ \\
\hline (Ii) IgG Positive & $1(1.6)$ \\
\hline (Iii) Both Positive & $8(13.1)$ \\
\hline
\end{tabular}




\section{PLATELET COUNT}

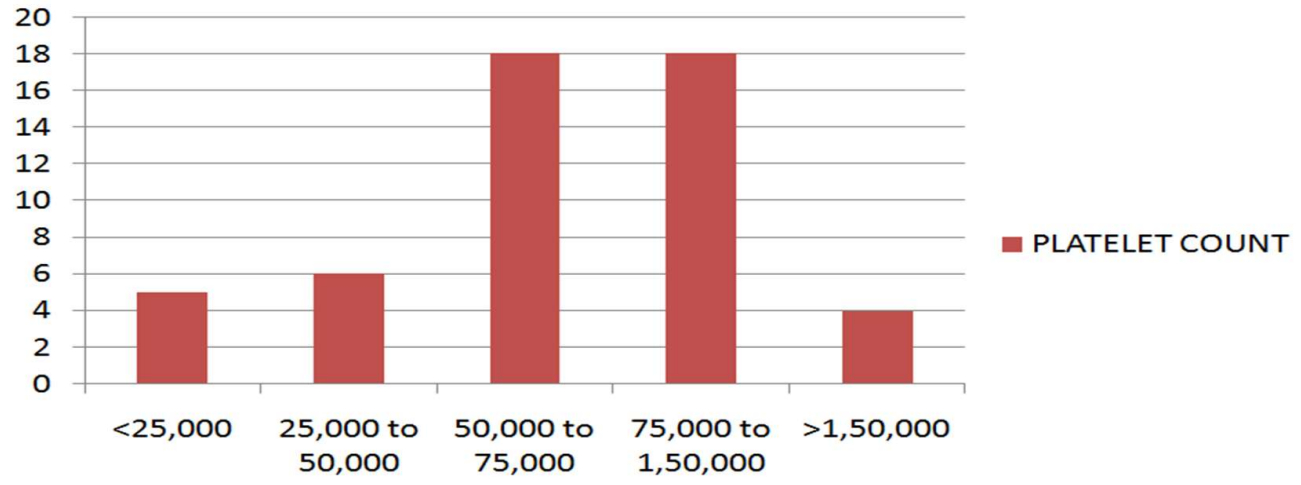

Fig-1: Platelet trend

Table-5: Comparison between severe and non-severe illness (symptoms)

\begin{tabular}{|c|c|c|c|c|c|}
\hline \multicolumn{2}{|c|}{ Parameters } & \multicolumn{2}{|c|}{ Severe illness $(n=22)$} & Non-severe & \multirow[t]{2}{*}{$P$ value $(<0.05)$} \\
\hline & & \multicolumn{2}{|c|}{$\mathrm{N}(\%)$} & N (\%) & \\
\hline \multirow[t]{2}{*}{ Sex } & Boys & $18(\varepsilon$ & & $18(46.2)$ & \multirow[t]{2}{*}{$0.007 *$} \\
\hline & Girls & $4(1$ & & $21(53.8)$ & \\
\hline \multirow[t]{2}{*}{ Duration of stay } & $\leq 5$ days & $3(1$ & & $31(79.5)$ & $<0.001^{*}$ \\
\hline & $>5$ days & $19(8$ & & $8(20.5)$ & $<0.001^{*}$ \\
\hline \multicolumn{2}{|c|}{ Headache } & \multicolumn{2}{|c|}{$11(50)$} & $15(38.5)$ & 0.382 \\
\hline \multicolumn{2}{|c|}{ Prodromal symptoms } & \multicolumn{2}{|c|}{$7(31.8)$} & $16(41)$ & 0.881 \\
\hline \multicolumn{2}{|c|}{ Fever } & \multicolumn{2}{|c|}{$21(95.5)$} & $38(97.4)$ & 0.676 \\
\hline \multirow{2}{*}{\multicolumn{2}{|c|}{ Duration of fever }} & $<3$ days & $3(13.6)$ & $15(38.5)$ & \multirow[t]{2}{*}{0.165} \\
\hline & & $\geq 3$ days & $19(86.4)$ & $24(61.5)$ & \\
\hline \multirow[t]{2}{*}{ Grading of fever } & Mild & $15(6$ & & $25(64.1)$ & \multirow[t]{2}{*}{0.436} \\
\hline & Moderate to high* & $7(3$ & & $14(35.9)$ & \\
\hline \multicolumn{2}{|c|}{ Chills and rigor } & \multicolumn{2}{|c|}{$5(22.7)$} & $8(20.5)$ & 0.839 \\
\hline \multicolumn{2}{|c|}{ Rashes* } & \multicolumn{2}{|c|}{$10(45.5)$} & $3(7.7)$ & $0.001 *$ \\
\hline \multicolumn{2}{|c|}{ Flushing } & \multicolumn{2}{|c|}{$17(77.3)$} & $8(20.5)$ & $<0.001 *$ \\
\hline \multicolumn{2}{|c|}{ Itching } & \multicolumn{2}{|c|}{$13(59.1)$} & $10(25.6)$ & $0.010^{*}$ \\
\hline \multicolumn{2}{|c|}{ Vomiting } & \multicolumn{2}{|c|}{$15(68.2)$} & $18(46.2)$ & 0.097 \\
\hline \multicolumn{2}{|c|}{ Loose stool } & \multicolumn{2}{|c|}{$3(13.6)$} & $4(10.3)$ & 0.691 \\
\hline \multicolumn{2}{|c|}{ Arthralgia } & \multicolumn{2}{|c|}{$11(50)$} & $15(38.5)$ & 0.382 \\
\hline \multicolumn{2}{|c|}{ Myalgia } & \multicolumn{2}{|c|}{$11(50)$} & $18(46.2)$ & 0.773 \\
\hline \multicolumn{2}{|c|}{ Anorexia } & \multicolumn{2}{|c|}{$19(86.4)$} & $17(43.6)$ & $0.001 *$ \\
\hline Abdon & lal pain & $14(6$ & & $14(35.9)$ & $0.037^{*}$ \\
\hline
\end{tabular}

Of the total 61 children enrolled in this study, only five children diagnosed with severe dengue, and 17 had dengue with warning signs. These two categories were combined to form a single severe illness group and compared with the remaining who had either dengue fever or probable dengue (non-severe illness group). Among the critical illness groups, 18 were boys. Nineteen children belonging to the severe illness group stayed in the hospital for more than five days. Flushing and the rashes were significantly more common in the severe illness group. Similarly, anorexia, abdominal pain, edema and itching were more frequent in the critical illness group. However, no significant difference was noted between the two groups in the occurrence of other symptoms (chills, vomiting, loose stool, headache, arthralgia and myalgia). 
Table-6: Comparison between children having a severe and non-severe illne (signs and laboratory parameters)

\begin{tabular}{|c|c|c|c|c|}
\hline \multirow{2}{*}{$\begin{array}{c}\text { Heart } \\
\text { rate }\end{array}$} & Tachycardia & $4(18.2)$ & 0 & \multirow{2}{*}{$0.015^{*}$} \\
\hline \multicolumn{2}{|c|}{ Bradycardia } & $4(18.2)$ & $5(12.8)$ & \\
\hline Tachypnea & $4(18.2)$ & 0 & $0.006^{*}$ \\
\hline Hypotension & $2(9.1)$ & 0 & 0.056 \\
\hline HESS test positivity & $15(68.2)$ & $3(7.7)$ & $<0.001^{*}$ \\
\hline Edema & $7(31.8)$ & $2(5.1)$ & $0.005^{*}$ \\
\hline Oliguria & $4(18.2)$ & 0 & $0.006^{*}$ \\
\hline Hepatomegaly & $16(72.7)$ & $21(53.8)$ & 0.080 \\
\hline Anemia & $5(22.7)$ & $19(48.7)$ & $0.033^{*}$ \\
\hline Severe thrombocytopenia & $14(63.6)$ & $7(17.9)$ & $<0.001^{*}$ \\
\hline Leukopenia & $18(81.8)$ & $26(66.7)$ & 0.205 \\
\hline Na $<\mathbf{1 3 5}$ Meq/L & $5(22.7)$ & 0 & $0.002^{*}$ \\
\hline SGOT(>120 IU/L) & $11(50)$ & $14(35.9)$ & 0.788 \\
\hline Hypoalbuminemia (<2.5 gm/dI) & $5(22.7)$ & 0 & $0.002^{*}$ \\
\hline Hydrops gall bladder & $1(4.5)$ & $2(5.1)$ & 0.631 \\
\hline
\end{tabular}

Among the clinical sign tachycardia (disproportionate to the degree of fever), tachypnea and oliguria were seen only in the severe illness group. The occurrence of bradycardia and hepatomegaly were similar in the two groups. Hyponatremia and low albumin noticed in $5(22.7 \%)$ children who had severe illness. Elevated liver enzyme and leucopenia were distributed similarly in the two group.

\section{Discussion}

Boys were more commonly affected in this study group and they also had higher tendency to have severe illness. Similar male preponderance was noted in other studies which may be attributed to the presence of more exposed area leading to more significant chance of mosquito-borne diseases [15,16]. Majority of the children belonged to the 6-12 age group which was also reported in other studies $[15,17]$.

Fever was the most common symptom. Vomiting and anorexia were also commonly seen in our patients. Other widelyrecognisedsymptoms were arthralgia, abdomen pain and headache. This symptom pattern is similar to those reported in several studies [17, 18]. Tourniquet test was positive only in few cases. Similar results observed in other Indian studies, probably due to the darker complexion of the study patients here [19, 20] However, it was more frequently seen in children with severe illness. Similarly, flushing was also seen more commonly in children with severedisease. These features can be used as an early marker to identify such children. More than a third of the children in our study reported itching during the recovery period. Some of them also had an erythematous rash which was different from the rashes noticed during the initial part of the illness. This rash was not blanchable, more confluent with islets of healthy skin which has been describedas "white islands in a sea of red" $[21,22]$.
One-tenth of the total cases had bleeding manifestations with petechiae being the most common manifestation. A similar result was noticed in the study conducted by Mishraet al [19]. Nearly one-third of children in our study had prodromal symptoms like a cough and runny nose. Other studies did not report these symptoms. The difference could be because this study was conducted during an epidemic of dengue infections, and there was a high index of suspicion among health professionals to screen for dengue. Similar illness presenting with a cough and runny nose during the non-epidemic season would have been regarded as non-specific viral illnesses. More than half of the children [57\%] in this study had hepatomegaly similar to previous reviews [23]. However, two children had hepato splenomegaly. Both of them were found to have malariaco-infection.

Thereby suggesting that in our setting whenever there is associated splenomegaly in children with dengue, coinfections with other vector-borne diseases that share similar seasonal trends like malaria and scrub typhus should be considered [24]. Apart from patients with malarial co-infection, two other patient had persistent fever spikes lasting for more than 7 days. These children were found to have gram negative bacterial sepsis complicating their underlying dengue infection, highlighting the need for evaluating children with persistent fever to look for co-infections. 
Among the various laboratory parameters, thrombocytopenia was the most frequently noticed abnormality. Majority of them had mild to moderate thrombocytopenia, and a small proportion showed normal platelet counts. Almost three fourth of the children had leucopenia $[20,25]$. Isolated elevation of SGOT $(>120$ IU/L) was present in $40 \%$ of the children in our study. A similar pattern of transaminitis was described in previous studies, and they observed a positive correlation between the level of SGOT and the severity of illness [26]. Hyponatremia and low albumin noted in children with severe disease, highlighting the importance of these parameters in assessing the risk of severe disease.

\section{Conclusion}

Dengue fever is a common cause of acute febrile illness, especially during monsoon season. Though the manifestations of dengue are similar to other viral infections, the disease severity and mortality is more in dengue, thus requiring early diagnosis. As observed in our study the presence of prodromal respiratory symptoms does not preclude the diagnosis of dengue. The course and severity of the illness are highly variable. Apart from the described warning signs, flushing, positive tourniquet test and laboratory derangements like hyponatremia and hypoalbuminemia can be considered as markers of severe illness. The presence of splenomegaly should raise the suspicion of co-infection if there is an unusual persistence of fever considering their common epidemiological background.

Clinical implications of this study- This study highlights the importance of suspecting and evaluating patients for dengue even if they present with prodromal respiratory symptoms and also highlights the need to screen for co-infections if the disease course is atypical.

\section{Contributions}

- Dr. Anand Ramakrishnan and Dr. Selvakumar- Data collection and analysis

- Dr. Benjamin Sagayaraj and Dr. BalammaSujathamanuscript preparation

- Dr. Porchelvan- Statistical analysis

Funding: Nil, Conflict of interest: None initiated, Perission from IRB: Yes

\section{References}

1. Guzman MG, Harris E. Dengue. Lancet. 2015 Jan 31; 385 (9966):453-65. doi: 10.1016/S0140-6736(14) 60572-9. Epub 2014 Sep 14.
2. Verhagen LM, de Groot R. Dengue in children. J Infect. 2014 Nov;69 Suppl 1:S77-86. doi: 10.1016/j. jinf. 2014. 07.020. Epub 2014 Sep 13.

3. Bhatt S, Gething PW, Brady OJ, Messina JP, Farlow AW, Moyes CL, Drake JM, Brownstein JS, Hoen AG, Sankoh O, Myers MF, George DB, Jaenisch T, Wint GR, Simmons CP, Scott TW, Farrar JJ, Hay SI. The global distribution and burden of dengue. Nature. 2013 Apr 25; 496 (7446): 504-7. doi: 10.1038/ nature 12060. Epub 2013 Apr 7.

4. Mutheneni SR, Morse AP, Caminade C, Upadhyayula SM. Dengue burden in India: recent trends and importance of climatic parameters. Emerg Microbes Infect. 2017 Aug 9; 6(8):e70.doi: 10.1038 / emi. 2017.57.

5.Kakkar M.Dengue fever is massively under-reported in India, hampering our response.BMJ. 2012 Dec 19; 345: e8574. doi: 10.1136/bmj.e8574.

6. Chakravarti A, Arora R, Luxemburger C. Fifty years of dengue in India. Trans R Soc Trop Med Hyg. 2012 May; 106 (5):273-82. doi: 10.1016/j.trstmh.2011. 12. 007. Epub 2012 Feb 21.

7. Hermann LL, Gupta SB, Manoff SB, Kalayanarooj $\mathrm{S}$, Gibbons RV, Coller BA. Advances in the under standing, management, and prevention of dengue. J Clin Virol. 2015 Mar;64:153-9. doi: 10.1016/j. jcv.2014. 08. 031. Epub 2014 Oct 20.

8. Mørch K, Manoharan A, Chandy S, Chacko N, Alvarez-Uria G, Patil S, et al. Acute undifferentiated fever in India: a multicentre study of aetiology and diagnostic accuracy. BMC Infect Dis. 2017 Oct 4; 17 (1): 665.doi: 10.1186/s12879-017-2764-3.

9. Trang NTH, Long NP, Hue TTM, Hung LP, Trung TD, Dinh DN, Luan NT, Huy NT, Hirayama K. Association between nutritional status and dengue infection: a systematic review and meta-analysis. BMC Infect Dis. 2016 Apr 20;16:172. doi: 10.1186/s12879016-1498-y.

10. Hansen C, Paintsil E.Infectious Diseases of Poverty in Children: A Tale of Two Worlds.Pediatr Clin North Am. 2016 Feb;63(1):37-66. doi: 10.1016/j. pcl. 2015.08.002.

11. Daudé É, Mazumdar S, Solanki V. Widespread fear of dengue transmission but poor practices of dengue prevention: A study in the slums of Delhi, India. PLoS One. 2017 Feb 10;12(2):e0171543. doi: 10.1371/ journal. pone. 0171543. eCollection 2017. 
12. Giersing BK, Modjarrad K, Kaslow DC, Moorthy VS; WHO Product Development for Vaccines Advisory Committee; WHO Product Development for Vaccines Product Development Advisory Committee:. Report from the World Health Organization's Product Development for Vaccines Advisory Committee (PDVAC) meeting, Geneva, 7-9th Sep 2015. Vaccine. 2016 Jun 3;34(26):2865-2869. doi: 10.1016/j. vaccine. 2016. 02.078. Epub 2016 Mar 15.

13. Malisheni M Khaiboullina SF, Rizvanov AA Takah N, Murewanhema G, Bates M. Clinical Efficacy, Safety, and Immunogenicity of a Live Attenuated Tetravalent Dengue Vaccine (CYD-TDV) in Children: A Systematic Review with Meta-analysis. Front Immunol.2017 Aug 4;8:863. doi: 10.3389/fimmu. 2017. 00863. eCollection 2017.

14. Guidelines on Clinical Management of Dengue Fever and Dengue Haemorrhagic Fever. The government of India. Directorate of National Vector Borne Diseases Control Programme. Directorate General of Health Services, Ministry of Health and Family Welfare. National Vector Borne Disease Control Programme (NVBDCP).

15. Pothapregada S, Kamalakannan B, Thulasingham M, Sampath S. Clinically Profiling Pediatric Patients with Dengue. J Glob Infect Dis. 2016 Jul-Sep;8(3):11520. doi: 10.4103/0974-777X.188596.

16. Sharma NL, Balasubramanyam V, Kandati J, Ponugoti M. Clinical and laboratory profile of dengue fever in children during an outbreak - one year study at tertiary care hospital, Chennai, Tamilnadu, India. Int J Contemp Pediatr2017;4:110-5.doi: 10.4103/ijmr.IJMR_ 1325_16.

17. Ramabhatta S, Palaniappan S, Hanumantharayappa N, Begum SV. The Clinical and Serological Profile of Pediatric Dengue. Indian J Pediatr.2017 Dec; 84 (12): 897-901. doi:10.1007/s12098-017-2423-0. Epub 2017 Sep 8.

18. Aggarwal A, Chandra J, Aneja S, Patwari AK, Dutta AK. An epidemic of dengue hemorrhagic fever and dengue shock syndrome in children in Delhi. Indian Pediatr. 1998 Aug;35(8):727-32.

19. Mishra S, Ramanathan R, Agarwal SK. Clinical profile of dengue fever in children: a study from Southern Odisha, India. Scientifica. 2016.doi: 10. 1155 / 2016/6391594.

20. Kalayanarooj S, Vaughn DW, Nimmannitya S, Green S, Suntayakorn S, Kunentrasai N, Viramitrachai W, Ratanachu-eke S, Kiatpolpoj S, Innis BL, Rothman AL, Nisalak A, Ennis FA. Early clinical and laboratory indicators of acute dengue illness. J Infect Dis. 1997 Aug;176(2):313-21.

21. ABM Shahidul Alam, S Anwar Sadat, Zakaria Swapanet al. Clinical Profile of Dengue Fever in Children Bangladesh J child health 2009;33(2),55-8. doi: 10.3329/bjch.v33i2.5678.

22. Vijayalakshmi AM, Jayavardhana A. Febrile rash and convalescent rash of dengue fever. Indian Pediatr. 2013 Jul;50(7):717.

23. Sahana KS1, Sujatha R. Clinical profile of dengue among children according to revised WHO classification: analysis of a 2012 outbreak from Southern India. Indian J Pediatr. 2015 Feb; 82(2):10913. doi: 10.1007/s12098-014-1523-3.

24. PS Sahu, M Sahu, S Ambuet al. A review of concurrent infections of malaria and dengue in Asia. Asian Pac J Trop Biomed 6,2016 july ;6(7):633-8. doi: 10.1016/j.apjtb.2016.05.008.

25. Ahmed S, Arif F, Yahya Y, Rehman A, Abbas $\mathrm{K}$, Ashraf S, Akram DS. Dengue fever outbreak in Karachi 2006--a study of profile and outcome of children under 15 years of age. J Pak Med Assoc. 2008 Jan; 58 (1):4-8.

26. Hari Kishan Jayanthi,Sai Krishna Tulasi. Correlation study between platelet count, leukocyte count, non-hemorrhagic complications, and duration of hospital stay in dengue fever with thrombocytopenia. J Family Med Prim Care. 2016 Jan-Mar; 5(1): 120-3. doi:10.4103/2249-4863.184635.

\section{How to cite this article?}

Anand R, Selvakumar L, Sagayaraj B, Sujatha B, Porchelvan. A study of clinical and laboratory profiles of dengue fever in children. Int J Pediatr Res. 2018;5(4):230-236.doi:10.17511/ijpr.2018.i04.12. 